\title{
OPTIMAL REGULARITY OF HARMONIC MAPS FROM A RIEMANNIAN MANIFOLD INTO A STATIC LORENTZIAN MANIFOLD
}

\section{TAKeSHI IsOBE}

\begin{abstract}
In this paper, we give an optimal regularity result for some class of weakly harmonic maps from a Riemannian manifold $M$ into a static Lorentzian manifold. Our main result is the following: For such class of weakly harmonic map $w$, there exists closed set $\Sigma \subset M$ such that $w$ is $C^{\infty}$ in $M \backslash \Sigma$ and the Hausdorff dimension of $\Sigma$ is less than or equal to $\operatorname{dim} M-3$.
\end{abstract}

\section{Introduction.}

In this paper, we study regularity of harmonic maps from a Riemannian manifold into a static Lorentzian manifold.

By definition, $N$ is a static Lorentzian manifold if and only if the following hold (see $[\mathbf{1 0}]$ ):

(i) $\quad N$ is the form $N=N_{0} \times \mathbf{R}$, where $N_{0}$ is a Riemannian manifold with a metric $g_{0}$.

(ii) The metric $g$ of $N$ is given by

$$
g\left(\left(\begin{array}{c}
\xi \\
\tau
\end{array}\right),\left(\begin{array}{c}
\xi^{\prime} \\
\tau^{\prime}
\end{array}\right)\right)=g_{0}\left(\xi, \xi^{\prime}\right)-\beta(x) \tau \tau^{\prime}
$$

for $\left(\begin{array}{c}\xi \\ \tau\end{array}\right),\left(\begin{array}{c}\xi^{\prime} \\ \tau^{\prime}\end{array}\right) \in T_{(x, t)} N=T_{x} N_{0} \times \mathbf{R}$, where $\beta: N_{0} \rightarrow \mathbf{R}^{+}$is a smooth positive function.

In such a case, we write $N=N_{0} \times{ }_{\beta} \mathbf{R}$.

In this paper we consider the case where $N_{0}$ is compact. We may assume, by Nash-Moser theorem, $N_{0}$ is a submanifold of $\mathbf{R}^{k}$ for some $k>1$. By the compactness of $N_{0}$, there exist constants $\beta_{\min }, \beta_{\max }>0$ such that $\beta_{\min } \leq$ $\beta(x) \leq \beta_{\max }$ for all $x \in N_{0}$.

Let $M$ be a Riemannian manifold with non-empty boundary $\partial M$. For a map $w=(u, t): M \rightarrow N_{0} \times{ }_{\beta} \mathbf{R}$, we define the energy $\mathcal{E}(w)$ of $w$ by:

$$
\mathcal{E}(w)=\mathcal{E}(u, t)=\int_{M}|\nabla u|^{2} d V-\int_{M} \beta(u)|\nabla t|^{2} d V
$$


where $d V$ is a volume measure on $M$.

By definition, $w=(u, t) \in H^{1}\left(M ; N_{0}\right) \times H^{1}(M ; \mathbf{R})$ is a (weakly) harmonic map if the following holds:

Since $N_{0}$ is compact, there exists a tubular neighborhood $\mathcal{O}$ of $N_{0}$ in $\mathbf{R}^{k}$ such that the nearest point projection $\Pi: \mathcal{O} \rightarrow N_{0}$ is a smooth fibration. For any $\phi \in C_{0}^{\infty}\left(M ; \mathbf{R}^{k}\right)$ and $\zeta \in C_{0}^{\infty}(M ; \mathbf{R})$, there holds:

$$
\left.\frac{d}{d \epsilon}\right|_{\epsilon=0} \mathcal{E}(\Pi \circ(u+\epsilon \phi), t+\epsilon \zeta)=0 .
$$

This means that $w=(u, t)$ is a stationary point with respect to the variation of the target manifold.

The weakly harmonic map $w=(u, t)$ satisfies the following equations in the distributional sense (see [8]):

$$
\left\{\begin{array}{ll}
\Delta u+A(u)(\nabla u, \nabla u)+\frac{1}{2} \nabla \beta(u)|\nabla t|^{2}=0 & \text { in } \quad M \\
\operatorname{div}(\beta(u) \nabla t)=0 & \text { in } \quad M
\end{array} .\right.
$$

Here, $A$ is the 2 nd fundamental form of the embedding $N_{0} \hookrightarrow \mathbf{R}^{k}$.

We consider Equation (1.1) with the prescribed boundary condition on $\partial M$ :

$$
w=(u, t)=(\varphi, \iota) \text { on } \partial M,
$$

where $(\varphi, \iota): \partial M \rightarrow N \times{ }_{\beta} \mathbf{R}$ is a given smooth map.

When the target manifold is a Riemannian, there are many regularity theories. For example, Schoen-Uhlenbeck [13] studied the regularity of minimizing harmonic mappings. In [5], [6] and [7], Hélein studied the regularity of weakly harmonic mappings when the dimension of the base manifold is 2. In [1], Bethuel studied the regularity of stationary harmonic mappings. In general, weakly harmonic maps are very singular. In [11], Riviere constructed weakly harmonic mapping from $B^{m}$ to $S^{n}(m \geq 3, n \geq 2)$ whose singular set is $B^{m}$. So there is no regularity theory for general weakly harmonic maps.

On the other hand, if the target manifold is a Lorentzian manifold, in [4], Greco constructed a smooth harmonic map when target is static Lorentzian and domain manifold is two dimensional. In [8], the author proved that any weakly harmonic map from 2-dimensional Riemannian manifold into static Lorentzian manifold is smooth and regularity results for some classes of harmonic maps when target is a static Lorentzian and the dimension of domain manifold is greater than 2 . 
More precisely, in [8], we considered the following class of solutions when $\operatorname{dim} M \geq 3$ :

For a given $u \in H_{\varphi}^{1}\left(M ; N_{0}\right)\left(H_{\varphi}^{1}\left(M ; N_{0}\right)\right.$ is the space of all $H^{1}\left(M ; N_{0}\right)$ maps with boundary value $\varphi$ on $\partial M$.), there exists a unique solution $t=t(u)$ for the 2nd equation of (1.1) with $t=\iota$ on $\partial M$.

Define the functional $\mathcal{F}: H_{\varphi}^{1}\left(M ; N_{0}\right) \rightarrow \mathbf{R}$ by

$$
\mathcal{F}(u)=\int_{M}|\nabla u|^{2} d V-\int_{M} \beta(u)|\nabla t(u)|^{2} d V
$$

It is shown in [8] that $\mathcal{F}$ is bounded from below in $H_{\varphi}^{1}\left(M ; N_{0}\right)$ and $\mathcal{F}$ attains the $\inf _{u \in H_{\varphi}^{1}\left(M ; N_{0}\right)} \mathcal{F}(u)$ for some $u \in H_{\varphi}^{1}\left(M ; N_{0}\right)$ and $(u, t(u))$ solves the Equations (1.1) and (1.2) in a weak sense. In this paper, we call such solutions as minimal type.

The main result of [8] is the following:

Theorem $1.1([8])$. Let $(u, t) \in H^{1}\left(M ; N_{0}\right) \times H^{1}(M ; \mathbf{R})$ be a minimal type solution. Then there exists a closed set $\Sigma \subset M$ such that $(u, t)$ is $C^{\infty}$ in $M \backslash \Sigma$. Moreover $\mathcal{H}^{m-2}(\Sigma)=0$, where $\mathcal{H}^{m-2}$ is the $(m-2)$-dimensional Hausdorff measure and $m=\operatorname{dim} M$.

Minimal type solutions correspond to minimizing harmonic maps when target manifold is a Riemannian. (Note that there are no energy minimizing harmonic mappings when target manifold is a Lorentzian since $\inf \mathcal{E}_{(u, t) \in H_{\varphi}^{1}\left(M ; N_{0}\right) \times H_{\iota}^{1}(M ; \mathbf{R})}(u, t)=-\infty$.)

In fact, when $\beta \equiv$ const., $(u, t)$ is a minimal type harmonic map if and only if $u \in H_{\varphi}^{1}\left(M ; N_{0}\right)$ is a Dirichlet energy minimizing map and $t$ is a harmonic function.

Also in such a case, by the regularity result of Schoen-Uhlenbeck [13], Theorem 1.1 may be improved.

However, there is a strong difference between $\mathcal{F}$-minimizing problem and Dirichlet energy minimization problem as in the Riemannian case. That is, the functional $\mathcal{F}$ is not a local functional. For example, $\mathcal{F}$-minimizer in $H_{\varphi}^{1}\left(M ; N_{0}\right)$ does not have a local minimizing property. This non-localness comes from the fact that $t(u)$ is implicitly defined in $M$ by $u$ and $\iota$ as a solution of the equation $\operatorname{div}(\beta(u) \nabla t(u))=0$ with $t(u)=\iota$ on $\partial M$. So we can not directly localize the problem. Since regularity problem is mainly local in the domain, this causes problems. This is the troublesome point in our problem.

However, in general case, as the above special case suggests, it is reasonable to conjecture that the size of the singular set $\Sigma$ in Theorem 1.1 is $\operatorname{dim} \Sigma \leq m-3$. 
In this paper we prove such conjecture is true. Our main result is the following:

Theorem A. Let $N=N_{0} \times{ }_{\beta} \mathbf{R}$ be a static Lorentzian manifold. Let $(u, t) \in H_{\varphi}^{1}\left(M ; N_{0}\right) \times H_{\iota}^{1}(M ; \mathbf{R})$ be a minimal type harmonic map. Then there exists a closed set $\Sigma \subset M$ such that $(u, t)$ is $C^{\infty}$ in $M \backslash \Sigma$. Moreover the Hausdorff dimension of $\Sigma$ is less than or equal to $m-3$ when $m \geq 3$. When $m=3, \Sigma$ is a discrete set and when $m=2, \Sigma$ is empty.

Remark 1.2. (a) The above singular set estimate is optimal. In fact, $u_{0}=x /|x|: B^{3} \rightarrow S^{2}$ is a Dirichlet energy minimizing map (see [2]) and $w=\left(u_{0}, t_{0}\right)$, where $t_{0} \equiv$ const. defines a minimal type harmonic map which is singular at $0 \in B^{3}$. Here $B^{3}=\left\{x \in \mathbf{R}^{3}:|x| \leq 1\right\}$. The examples of higher dimensional cases are constructed in the same way.

(b) Since $t(u)$ satisfies the elliptic equation $\operatorname{div}(\beta(u) \nabla t(u))=0$ with $0<$ $\beta_{\min } \leq \beta(u(x)) \leq \beta_{\max }<+\infty, t(u)$ is always Hölder continuous in $M$ by De Giorgi-Nash theorem [9].

(c) If $\operatorname{dim} M=2$, in [8], we proved that any weakly harmonic map in $H^{1}\left(M ; N_{0}\right) \times H^{1}(M ; \mathbf{R})$ is smooth in $M$.

The crucial step in the proof of Theorem A is a derivation of the monotonicity inequality. Here new difficulties arise due to the fact that the functional $\mathcal{F}$ is not a local functional as stated above. Thus it turns out that we need to analyze the behavior of solutions of some elliptic equations under the deformations of the domain manifold. These are carried out in $\S 2$.

Next step consists of compactness result for the families of scaled maps. As stated above, there is non sense to consider "local minimizing" maps in order to study regularity properties of (global) minimizing maps (since global $\mathcal{F}$-minimizer is not in general local $\mathcal{F}$-minimizer), so we need to consider compactness properties of scaled maps of global minimizers (which has, in general, no minimizing properties) in order to study local properties of (global) minimizing maps.

Finally, using Federer's dimension reduction argument [3], [12], [13], we obtain Theorem A. These are carried out in $\S 3$.

Acknowledgment. The author wishes to thank referee for his valuable comments and suggestions on a first version of this paper.

\section{Monotonicity inequality.}

In this section, we derive the monotonicity inequality for $\mathcal{F}$-minimizing map $u$ in $H_{\varphi}^{1}\left(M ; N_{0}\right)$. For simplicity, we consider the case $M=\Omega$ is flat, that is, $M$ is the open bounded set $\Omega$ in $\mathbf{R}^{m}$. The general case is more involved, but essentially the same method is applicable with slight modifications. 
Our main result is the following:

Proposition 2.1. Let $u \in H_{\varphi}^{1}\left(\Omega ; N_{0}\right)$ be a $\mathcal{F}$-minimizing map. For $a \in \Omega$ and $0<R_{1} \leq R_{2}<1 / 2 \operatorname{dist}(a, \partial \Omega)$, there exist constants $c, \alpha>0$ (depending only on $\beta$ and $m$ ) such that the following holds:

$$
\begin{aligned}
& R_{1}^{2-m} \int_{B_{R_{1}(a)}}|\nabla u|^{2} d x+2 \int_{R_{1} \leq|x-a| \leq R_{2}}|x-a|^{2-m}\left|\frac{\partial u}{\partial r}\right|^{2} d x \\
& \leq R_{2}^{2-m} \int_{B_{R_{2}}(a)}|\nabla u|^{2} d x+c\left(R_{1}^{\alpha}+R_{2}^{\alpha}\right) .
\end{aligned}
$$

Here $B_{\rho}(a)=\left\{x \in \mathbf{R}^{m}:|x-a|<\rho\right\}$.

Proof. Fix $\phi \in C_{0}^{\infty}(\Omega)$. For $|\epsilon|$ small, we set $\phi_{\epsilon}(x)=x+\epsilon \phi(x)$. For small $|\epsilon|, \phi_{\epsilon}: \Omega \rightarrow \Omega$ defines a diffeomorphism.

Let $u \in H_{\varphi}^{1}\left(M ; N_{0}\right)$ be a $\mathcal{F}$-minimizer. We set $u_{\epsilon}=u \circ \phi_{\epsilon}^{-1}$. For small $|\epsilon|$, $u_{\epsilon} \in H_{\varphi}^{1}\left(\Omega ; N_{0}\right)$.

We study the dependence of $\mathcal{F}\left(u_{\epsilon}\right)$ in $\epsilon$.

By some calculation, we obtain:

$$
\begin{aligned}
& \int_{\Omega}\left|\nabla u_{\epsilon}\right|^{2} d x \\
& =\int_{\Omega}|\nabla u|^{2} d x+\epsilon\left(\int_{\Omega}|\nabla u|^{2} \operatorname{div} \phi d x-2 \sum_{\substack{1 \leq i, j \leq m \\
1 \leq l \leq k}} \int_{\Omega} \frac{\partial u^{l}}{\partial x_{i}} \frac{\partial u^{l}}{\partial x_{j}} \frac{\partial \phi_{i}}{\partial x_{j}} d x\right)+O\left(\epsilon^{2}\right) .
\end{aligned}
$$

Next we study $\int_{\Omega} \beta\left(u_{\epsilon}\right)\left|\nabla t\left(u_{\epsilon}\right)\right|^{2} d x$.

Here some difficulties arise due to the non-localness of $t(u)$ with respect to $u$. That is, $t\left(u_{\epsilon}\right)$ is defined implicitly in $\Omega$ by $u_{\epsilon}$ and $\iota$ as a solution of the equations

$$
\left\{\begin{aligned}
\operatorname{div}\left(\beta\left(u_{\epsilon}\right) \nabla t\left(u_{\epsilon}\right)\right)=0 & \text { in } \quad \Omega, \\
t\left(u_{\epsilon}\right)=\iota & \text { on } \quad \partial \Omega .
\end{aligned}\right.
$$

So the equality $t\left(u_{\epsilon}\right) \circ \phi_{\epsilon}=t(u)$ does not hold in general. Thus we need the analysis of the behavior of $t\left(u_{\epsilon}\right) \circ \phi_{\epsilon}$ with respect to $\epsilon$.

We compute, by the change of variable $x=\phi_{\epsilon}(y)$,

$$
\int_{\Omega} \beta\left(u_{\epsilon}(x)\right)\left|\nabla t\left(u_{\epsilon}\right)(x)\right|^{2} d x
$$




$$
=\int_{\Omega} \beta(u)\left|\nabla t\left(u_{\epsilon}\right)\left(\phi_{\epsilon}(y)\right)\right|^{2}(1+\epsilon \operatorname{div} \phi) d y+O\left(\epsilon^{2}\right) \int_{\Omega}\left|\nabla t\left(u_{\epsilon}\right)\left(\phi_{\epsilon}(y)\right)\right|^{2} d y .
$$

We set $\hat{t}_{\epsilon}:=t\left(u_{\epsilon}\right) \circ \phi_{\epsilon}$.

Since

$$
\nabla t\left(u_{\epsilon}\right)\left(\phi_{\epsilon}(y)\right)=J^{-1}\left(\phi_{\epsilon}(y)\right) \nabla \hat{t}_{\epsilon}(y),
$$

where $\nabla t\left(u_{\epsilon}\right)=\left(\frac{\partial t\left(u_{\epsilon}\right)}{\partial x_{1}}, \ldots, \frac{\partial t\left(u_{\epsilon}\right)}{\partial x_{m}}\right)^{t}, \nabla \hat{t}_{\epsilon}(y)=\left(\frac{\partial \hat{t}_{\epsilon}}{\partial y_{1}}, \ldots, \frac{\partial \hat{t}_{\epsilon}}{\partial y_{m}}\right)^{t}$ and $J\left(\phi_{\epsilon}\right)=$ jacobian of $\phi_{\epsilon}$, and

$$
J^{-1}\left(\phi_{\epsilon}(y)\right)=\left(\delta_{i j}-\epsilon \frac{\partial \phi_{j}}{\partial y_{i}}+O\left(\epsilon^{2}\right)\right)_{i j}
$$

we obtain

$$
\left|\nabla t\left(u_{\epsilon}\right)\left(\phi_{\epsilon}(y)\right)\right|^{2}=\left|\nabla \hat{t}_{\epsilon}(y)\right|^{2}-2 \epsilon \sum_{1 \leq i, j \leq m} \frac{\partial \hat{t}_{\epsilon}}{\partial y_{i}} \frac{\partial \hat{t}_{\epsilon}}{\partial y_{j}} \frac{\partial \phi_{j}}{\partial y_{i}}+O\left(\epsilon^{2}\right) R_{1}\left(\nabla \hat{t}_{\epsilon}, \phi\right) .
$$

Here $R_{1}\left(\nabla \hat{t}_{\epsilon}, \phi\right)$ is a quadratic form in $\nabla \hat{t}_{\epsilon}$.

Combining (2.2) and (2.3), we obtain:

$$
\begin{aligned}
& \int_{\Omega} \beta\left(u_{\epsilon}\right)\left|\nabla t\left(u_{\epsilon}\right)\right|^{2} d x \\
& =\int_{\Omega} \beta(u)\left\{\left|\nabla \hat{t}_{\epsilon}\right|^{2}-2 \epsilon \sum_{1 \leq i, j \leq m} \frac{\partial \hat{t}_{\epsilon}}{\partial y_{i}} \frac{\partial \hat{t}_{\epsilon}}{\partial y_{j}} \frac{\partial \phi_{j}}{\partial y_{i}}\right\}(1+\epsilon \operatorname{div} \phi) d y \\
& \quad+O\left(\epsilon^{2}\right) R_{2}\left(\nabla \hat{t}_{\epsilon}, \phi\right) \\
& =\int_{\Omega} \beta(u)\left|\nabla \hat{t}_{\epsilon}\right|^{2} d y+\epsilon\left(\int_{\Omega} \beta(u)\left|\nabla \hat{t}_{\epsilon}\right|^{2} \operatorname{div} \phi-2 \beta(u) \sum_{1 \leq i, j \leq m} \frac{\partial \hat{t}_{\epsilon}}{\partial y_{i}} \frac{\partial \hat{t}_{\epsilon}}{\partial y_{j}} \frac{\partial \phi_{j}}{\partial y_{i}} d y\right) \\
& \quad+O\left(\epsilon^{2}\right) R_{3}\left(\nabla \hat{t}_{\epsilon}, \phi\right) .
\end{aligned}
$$

Here $R_{2}\left(\nabla \hat{t}_{\epsilon}, \phi\right)$ and $R_{3}\left(\nabla \hat{t}_{\epsilon}, \phi\right)$ are quadratic functionals in $\nabla \hat{t}_{\epsilon}$.

Since $t\left(u_{\epsilon}\right)$ is a critical point of the functional $t \mapsto \int_{\Omega} \beta\left(u_{\epsilon}\right)|\nabla t|^{2} d x$, by (2.4), $\hat{t}_{\epsilon}$ satisfies the following for any $\Phi \in C_{0}^{\infty}(\Omega ; \mathbf{R})$ :

$$
\begin{aligned}
& \int_{\Omega} \beta(u) \nabla \hat{t}_{\epsilon} \cdot \nabla \Phi d y+\epsilon\left(\int_{\Omega} \beta(u) \nabla \hat{t}_{\epsilon} \cdot \nabla \Phi \operatorname{div} \phi d y\right. \\
& \left.\quad-\int_{\Omega} \beta(u) \sum_{1 \leq i, j \leq m} \frac{\partial \Phi}{\partial y_{i}} \frac{\partial \hat{t}_{\epsilon}}{\partial y_{j}} \frac{\partial \phi_{i}}{\partial y_{j}} d y-\int_{\Omega} \beta(u) \sum_{1 \leq i, j \leq m} \frac{\partial \hat{t}_{\epsilon}}{\partial y_{i}} \frac{\partial \Phi}{\partial y_{j}} \frac{\partial \phi_{i}}{\partial y_{j}} d y\right)
\end{aligned}
$$




$$
+O\left(\epsilon^{2}\right) R_{3}^{\prime}\left(\nabla \Phi, \nabla \hat{t}_{\epsilon}, \phi\right) .
$$

Here $R_{3}^{\prime}\left(\nabla \Phi, \nabla \hat{t}_{\epsilon}, \phi\right)$ is a linear form in $\nabla \Phi$ and $\nabla \hat{t}_{\epsilon}$.

By density, (2.5) holds for any $\Phi \in H_{0}^{1}(\Omega ; \mathbf{R})$. We take $\Phi=\hat{t}_{\epsilon}-t(u) \in$ $H_{0}^{1}(\Omega ; \mathbf{R})$. Then we have

$$
\begin{aligned}
& \int_{\Omega} \nabla \hat{t}_{\epsilon} \cdot \nabla\left(\hat{t}_{\epsilon}-t(u)\right) d y+\epsilon\left(\int_{\Omega} \beta(u) \nabla \hat{t}_{\epsilon} \cdot \nabla\left(\hat{t}_{\epsilon}-t(u)\right) \operatorname{div} \phi d y\right. \\
& -\int_{\Omega} \beta(u) \sum_{1 \leq i, j \leq m} \frac{\partial}{\partial y_{j}}\left(\hat{t}_{\epsilon}-t(u)\right) \frac{\partial \hat{t}_{\epsilon}}{\partial y_{j}} \frac{\partial \phi_{i}}{\partial y_{j}} d y \\
& \left.\quad-\int_{\Omega} \beta(u) \sum_{1 \leq i, j \leq m} \frac{\partial \hat{t}_{\epsilon}}{\partial y_{j}} \frac{\partial}{\partial y_{i}}\left(\hat{t}_{\epsilon}-t(u)\right) \frac{\partial \phi_{i}}{\partial y_{j}} d y\right) \\
& +O\left(\epsilon^{2}\right) R_{3}^{\prime}\left(\nabla\left(\hat{t}_{\epsilon}-t(u)\right), \nabla \hat{t}_{\epsilon}, \phi\right)=0 .
\end{aligned}
$$

On the other hand, since $\operatorname{div}(\beta(u) \nabla t(u))=0$, we have:

$$
\int_{\Omega} \beta(u) \nabla t(u) \cdot \nabla\left(\hat{t}_{\epsilon}-t(u)\right) d y=0 .
$$

Subtracting (2.7) form (2.6), we obtain:

$$
\int_{\Omega}\left|\nabla \hat{t}_{\epsilon}-\nabla t(u)\right|^{2} d y \leq C_{1}\left(|\epsilon|+\epsilon^{2}\right)\left\|\nabla \hat{t}_{\epsilon}\right\|_{L^{2}(\Omega)}\left\|\nabla \hat{t}_{\epsilon}-\nabla t(u)\right\|_{L^{2}(\Omega)},
$$

where $c_{1}>0$ is a constant which may depend on $\phi$ but does not depend on $\epsilon$.

We claim that $\hat{t}_{\epsilon}$ is bounded in $H^{1}$.

To prove this, first observe that by (2.4) there exists $c_{2}>0$ independent of $\epsilon$ with $|\epsilon|$ small such that

$$
\int_{\Omega}\left|\nabla \hat{t}_{\epsilon}\right|^{2} d y \leq c_{2} \int_{\Omega}\left|\nabla t\left(u_{\epsilon}\right)\right|^{2} d y
$$

Let $h$ be the harmonic extension of $\iota$ to $\Omega$, i.e.,

$$
\begin{cases}\Delta h=0 & \text { in } \quad \Omega, \\ h=\iota & \text { on } \quad \partial \Omega .\end{cases}
$$

Then by the minimizing property of $t\left(u_{\epsilon}\right)$, we obtain

$$
\int_{\Omega} \beta\left(u_{\epsilon}\right)\left|\nabla t\left(u_{\epsilon}\right)\right|^{2} d y \leq \int_{\Omega} \beta\left(u_{\epsilon}\right)|\nabla h|^{2} d y \leq \beta_{\max } \int_{\Omega}|\nabla h|^{2} d y .
$$


Therefore we have

$$
\int_{\Omega}\left|\nabla t\left(u_{\epsilon}\right)\right|^{2} d y \leq \frac{\beta_{\max }}{\beta_{\min }} \int_{\Omega}|\nabla h|^{2} d y=\text { independent of } \epsilon .
$$

This completes the proof of the claim.

Combining this claim with (2.8), we get

$$
\int_{\Omega}\left|\nabla \hat{t}_{\epsilon}-\nabla t(u)\right|^{2} d y \leq c_{3} \epsilon^{2}
$$

where $c_{3}$ is a constant independent of $\epsilon$.

We set $\hat{t}_{\epsilon}=t(u)+\alpha_{\epsilon}$. Then

$$
\left.\alpha_{\epsilon}\right|_{\partial \Omega}=0
$$

and

$$
\int_{\Omega}\left|\nabla \alpha_{\epsilon}\right|^{2} d y=O\left(\epsilon^{2}\right)
$$

By (2.4), we obtain

$$
\begin{aligned}
& \int_{\Omega} \beta\left(u_{\epsilon}\right)\left|\nabla t\left(u_{\epsilon}\right)\right|^{2} d x \\
& =\int_{\Omega} \beta(u)\left|\nabla t(u)+\nabla \alpha_{\epsilon}\right|^{2} d y+\epsilon\left(\int_{\Omega} \beta(u)\left|\nabla t(u)+\nabla \alpha_{\epsilon}\right|^{2} \operatorname{div} \phi d y\right. \\
& \left.\quad-2 \int_{\Omega} \beta(u) \sum_{1 \leq i, j \leq m}\left(\frac{\partial t(u)}{\partial y_{i}}+\frac{\partial \alpha_{\epsilon}}{\partial y_{i}}\right)\left(\frac{\partial t(u)}{\partial y_{j}}+\frac{\partial \alpha_{\epsilon}}{\partial y_{j}}\right) \frac{\partial \phi_{j}}{\partial y_{i}} d y\right)+O\left(\epsilon^{2}\right) \\
& =\int_{\Omega} \beta(u)|\nabla t(u)|^{2} d y+2 \int_{\Omega} \beta(u) \nabla t(u) \cdot \nabla \alpha_{\epsilon} d y+\int_{\Omega} \beta(u)\left|\nabla \alpha_{\epsilon}\right|^{2} d y \\
& \quad+\epsilon\left(\int_{\Omega} \beta(u)|\nabla t(u)|^{2} \operatorname{div} \phi d y+2 \int_{\Omega} \beta(u) \nabla t(u) \cdot \nabla \alpha_{\epsilon} \operatorname{div} \phi d y\right. \\
& \quad+\int_{\Omega} \beta(u)\left|\nabla \alpha_{\epsilon}\right|^{2} \operatorname{div} \phi d y \\
& \quad-2 \sum_{1 \leq i, j \leq m}\left\{\int_{\Omega} \beta(u) \frac{\partial t(u)}{\partial y_{i}} \frac{\partial t(u)}{\partial y_{j}} \frac{\partial \phi_{i}}{\partial y_{j}} d y+\int_{\Omega} \beta(u) \frac{\partial \alpha_{\epsilon}}{\partial y_{i}} \frac{\partial t(u)}{\partial y_{j}} \frac{\partial \phi_{i}}{\partial y_{j}} d y\right. \\
& \left.\left.\quad+\int_{\Omega} \beta(u) \frac{\partial t(u)}{\partial y_{i}} \frac{\partial \alpha_{\epsilon}}{\partial y_{i}} \frac{\partial \phi_{j}}{\partial y_{i}} d y+\int_{\Omega} \beta(u) \frac{\partial \alpha_{\epsilon}}{\partial y_{i}} \frac{\partial \alpha_{\epsilon}}{\partial y_{j}} \frac{\partial \phi_{i}}{\partial y_{j}} d y\right\}\right)+O\left(\epsilon^{2}\right) .
\end{aligned}
$$

Here, $\int_{\Omega} \beta(u) \nabla t(u) \cdot \nabla \alpha_{\epsilon} d y=0$ by $\operatorname{div}(\beta(u) \nabla t(u))=0$ and $(2.10)$.

Thus, by (2.11) and (2.12) we obtain

$$
\int_{\Omega} \beta\left(u_{\epsilon}\right)\left|\nabla t\left(u_{\epsilon}\right)\right|^{2} d x
$$




$$
\begin{aligned}
= & \int_{\Omega} \beta(u)|\nabla t(u)|^{2} d y+\epsilon\left(\int_{\Omega} \beta(u)|\nabla t(u)|^{2} \operatorname{div} \phi d y\right. \\
& \left.-2 \int_{\Omega} \beta(u) \sum_{1 \leq i, j \leq m} \frac{\partial t(u)}{\partial y_{i}} \frac{\partial t(u)}{\partial y_{j}} \frac{\partial \phi_{j}}{\partial y_{i}} d y\right)+O\left(\epsilon^{2}\right) .
\end{aligned}
$$

Combining (2.1) and (2.13) we get

$$
\begin{aligned}
\mathcal{F}\left(u_{\epsilon}\right)= & \int_{\Omega}|\nabla u|^{2} d x-\int_{\Omega} \beta(u)|\nabla t(u)|^{2} d x+\epsilon\left(\int_{\Omega}|\nabla u|^{2} \operatorname{div} \phi d x\right. \\
& -2 \sum_{\substack{1 \leq i \leq m \\
1 \leq l \leq k}} \int_{\Omega} \frac{\partial u^{l}}{\partial x_{i}} \frac{\partial u^{l}}{\partial x_{j}} \frac{\partial \phi_{j}}{\partial x_{i}} d x-\int_{\Omega} \beta(u)|\nabla t(u)|^{2} \operatorname{div} \phi d x \\
& \left.+2 \sum_{1 \leq i, j \leq m} \int_{\Omega} \beta(u) \frac{\partial t(u)}{\partial x_{i}} \frac{\partial t(u)}{\partial x_{j}} \frac{\partial \phi_{j}}{\partial x_{i}} d x\right)+O\left(\epsilon^{2}\right) \\
= & \mathcal{F}(u)+\epsilon\left(\int_{\Omega}|\nabla u|^{2} \operatorname{div} \phi d x-2 \sum_{\substack{1 \leq i, j \leq m \\
1 \leq l \leq k}} \int_{\Omega} \frac{\partial u^{l}}{\partial x_{i}} \frac{\partial u^{l}}{\partial x_{j}} \frac{\partial \phi_{j}}{\partial x_{i}} d x\right. \\
& -\int_{\Omega} \beta(u)|\nabla t(u)|^{2} \operatorname{div} \phi d x \\
& \left.+2 \sum_{1 \leq i, j \leq m} \int_{\Omega} \beta(u) \frac{\partial t(u)}{\partial x_{i}} \frac{\partial t(u)}{\partial x_{j}} \frac{\partial \phi_{j}}{\partial x_{i}} d x\right)+O\left(\epsilon^{2}\right) .
\end{aligned}
$$

Since $u_{\epsilon} \in H_{\varphi}^{1}\left(\Omega ; N_{0}\right)$, by the minimality of $u$ we have $\mathcal{F}(u) \leq \mathcal{F}\left(u_{\epsilon}\right)$ for small $|\epsilon|$.

Therefore by (2.14) we obtain

$$
\begin{aligned}
& \int_{\Omega}|\nabla u|^{2} \operatorname{div} \phi d x-2 \sum_{\substack{1 \leq i, j \leq m \\
1 \leq l \leq k}} \int_{\Omega} \frac{\partial u^{l}}{\partial x_{i}} \frac{\partial u^{l}}{\partial x_{j}} \frac{\partial \phi_{i}}{\partial x_{j}} d x \\
& -\int_{\Omega} \beta(u)|\nabla t(u)|^{2} \operatorname{div} \phi d x+2 \sum_{1 \leq i, j \leq m} \int_{\Omega} \beta(u) \frac{\partial t(u)}{\partial x_{i}} \frac{\partial t(u)}{\partial x_{j}} \frac{\partial \phi_{j}}{\partial x_{i}} d x=0
\end{aligned}
$$

for all $\phi \in C_{0}^{\infty}\left(\Omega ; \mathbf{R}^{n}\right)$.

Here we take, in particular, $\phi(x)=\zeta(r / \rho)(x-a)$, where $\zeta \in C^{\infty}(\mathbf{R})$ is such that $\zeta(r) \equiv 1$ for $r \leq 1 / 2, \zeta(r) \equiv 0$ for $r \geq 1, \zeta^{\prime} \leq 0$ and $\rho>0$ satisfies $B_{\rho}(a) \subset \subset \Omega$.

Then by some computations, we obtain

$$
-\rho \int_{\Omega}|\nabla u|^{2} \frac{d}{d \rho}\left\{\phi\left(\frac{r}{\rho}\right)\right\} d x+(m-2) \int_{\Omega}|\nabla u|^{2} \phi\left(\frac{r}{\rho}\right) d x
$$




$$
\begin{aligned}
& +2 \rho \int_{\Omega}\left|\frac{\partial u}{\partial r}\right|^{2} \frac{d}{d \rho}\left\{\phi\left(\frac{r}{\rho}\right)\right\} d x+\rho \int_{\Omega} \beta(u)|\nabla t(u)|^{2} \frac{d}{d \rho}\left\{\phi\left(\frac{r}{\rho}\right)\right\} d x \\
& -(m-2) \int_{\Omega} \beta(u)|\nabla t(u)|^{2} \phi\left(\frac{r}{\rho}\right) d x
\end{aligned}
$$

$$
-2 \rho \int_{\Omega} \beta(u)\left|\frac{\partial t(u)}{\partial r}\right|^{2} \frac{d}{d \rho}\left\{\phi\left(\frac{r}{\rho}\right)\right\} d x=0
$$

Letting $\phi$ approach to the characteristic function of $(-\infty, 1]$, we obtain

$$
\begin{aligned}
& -\rho \frac{d}{d \rho} \int_{B_{\rho}(a)}|\nabla u|^{2} d x+(m-2) \int_{B_{\rho}(a)}|\nabla u|^{2} d x \\
& +2 \rho \frac{d}{d \rho} \int_{B_{\rho}(a)}\left|\frac{\partial u}{\partial r}\right|^{2} d x+\rho \frac{d}{d \rho} \int_{B_{\rho}(a)} \beta(u)|\nabla t(u)|^{2} d x
\end{aligned}
$$

$$
-(m-2) \int_{B_{\rho}(a)} \beta(u)|\nabla t(u)|^{2} d x-2 \rho \frac{d}{d \rho} \int_{\Omega} \beta(u)\left|\frac{\partial t(u)}{\partial r}\right|^{2} d x=0 .
$$

(2.16) is equivalent to the following:

$$
\begin{aligned}
& \frac{d}{d \rho}\left\{\rho^{2-m} \int_{B_{\rho}(a)}|\nabla u|^{2} d x-\rho^{2-m} \int_{B_{\rho}(a)} \beta(u)|\nabla t(u)|^{2} d x\right\} \\
& =2 \rho^{2-m} \int_{\partial B_{\rho}(a)}\left|\frac{\partial u}{\partial r}\right|^{2} d x-2 \rho^{2-m} \int_{\partial B_{\rho}(a)} \beta(u)\left|\frac{\partial t(u)}{\partial r}\right|^{2} d x .
\end{aligned}
$$

Integrating (2.17) from $R_{1}$ to $R_{2}$ we obtain

$$
\begin{aligned}
& R_{2}^{2-m} \int_{B_{R_{2}}(a)}|\nabla u|^{2} d x-R_{1}^{2-m} \int_{B_{R_{1}}(a)}|\nabla u|^{2} d x \\
& \quad-R_{2}^{2-m} \int_{B_{R_{2}}(a)} \beta(u)|\nabla t(u)|^{2} d x+R_{1}^{2-m} \int_{B_{R_{1}(a)}} \beta(u)|\nabla t(u)|^{2} d x \\
& =2 \int_{R_{1} \leq|x-a| \leq R_{2}}|x-a|^{2-m}\left|\frac{\partial u}{\partial r}\right|^{2} d x \\
& \quad-2 \int_{R_{1} \leq|x-a| \leq R_{2}} \beta(u)|x-a|^{2-m}\left|\frac{\partial t(u)}{\partial r}\right|^{2} d x .
\end{aligned}
$$

Since $t(u)$ is a solution of the equation $\operatorname{div}(\beta(u) \nabla t(u))=0$ in $\Omega$ and $0<$ $\beta_{\min } \leq \beta(u) \leq \beta_{\max }<+\infty$, by De Giorgi-Nash theorem and p. 59, Lemma 
4.3 in [9], there exist constants $\alpha>0$ and $c>0$ depending only on $\beta_{\max }$, $\beta_{\min }$ and $m$ such that the following hold for $0<r<\operatorname{dist}(a, \partial \Omega)$ :

$$
\begin{gathered}
r^{2-m} \int_{B_{r}(a)}|\nabla t(u)|^{2} d x \leq c r^{\alpha}, \\
\int_{B_{r}(a)}|x-a|^{2-m}|\nabla t(u)|^{2} d x \leq c r^{\alpha} .
\end{gathered}
$$

Combining (2.18), (2.19) and (2.20), there exists constants $\alpha>0$ and $c>0$ depending only on $\beta$ and $m$ such that

$$
\begin{aligned}
& R_{1}^{2-m} \int_{B_{R_{1}}(a)}|\nabla u|^{2} d x+2 \int_{R_{1} \leq|x-a| \leq R_{2}}|x-a|^{2-m}\left|\frac{\partial u}{\partial r}\right|^{2} d x \\
& \leq R_{2}^{2-m} \int_{B_{R_{2}}(a)}|\nabla u|^{2} d x+c\left(R_{1}^{\alpha}+R_{2}^{\alpha}\right) .
\end{aligned}
$$

This completes the proof of Proposition 2.1.

\section{Proof of Theorem A.}

In this section, we prove our main theorem A. We recall the following two facts from $[8]$.

Lemma 3.1 ([8, Lemma 3.4]). Let $u \in H_{\varphi}^{1}\left(M ; N_{0}\right)$ be a $\mathcal{F}$-minimizer. Let $B_{r}(a)$ be a ball such that $B_{r}(a) \subset \subset \Omega$ and let $v \in H^{1}\left(B_{r}(a) ; N_{0}\right)$ with $v=u$ on $\partial B_{r}(a)$. Then there exists $\eta \in H_{\varphi}^{1}\left(M ; N_{0}\right)$ (depending on $u, v$, a and $r$ ) such that the following holds:

$$
\begin{aligned}
& \int_{B_{r}(a)}|\nabla u|^{2} d x-\int_{B_{r}(a)} \beta(u)|\nabla t(u)|^{2} d x \\
& \leq \int_{B_{r}(a)}|\nabla v|^{2} d x+\beta_{\max } \int_{B_{r}(a)}|\nabla t(\eta)|^{2} d x .
\end{aligned}
$$

The following small energy regularity theorem is proved in [8]:

Lemma 3.2 ([8, Lemma 3.10]). There exist $R_{0}>, \bar{\epsilon}>0, \gamma>0$ and $c>0$ such that if a $\mathcal{F}$-minimizing map $u \in H_{\varphi}^{1}\left(M ; N_{0}\right)$ satisfies $R^{2-m} \int_{B_{2 R}(a)}|\nabla u|^{2} d x<\bar{\epsilon}$ for some $R<\min \left\{R_{0}, \frac{1}{2} \operatorname{dist}(a, \partial \Omega)\right\}$, then $u$ is $\gamma$-Hölder continuous in $B_{R}(a)$ and the following holds for any $x, y \in B_{R}(a)$ :

$$
|u(x)-u(y)| \leq c|x-y|^{\gamma} .
$$

We begin the following: 
Proposition 3.3. Let $u \in H_{\varphi}^{1}\left(M ; N_{0}\right)$ be a $\mathcal{F}$-minimizing map. For a given $B>0$, there exists $\epsilon_{0}>, c>0, \delta>0$ and $\rho_{0}>0$ such that if $\rho<\rho_{0}, B_{\rho}(b) \subset$ $\subset \Omega(b \in \Omega), \rho^{2-n} \int_{B_{\rho}(b)}|\nabla u|^{2} d x \leq B$ and $\rho^{-n} \int_{B_{\rho}(b)}\left|u-(u)_{\rho, b}\right|^{2} d x \leq \epsilon_{0}$ $\left((u)_{\rho, b}=1 /\left|B_{\rho}(b)\right| \int_{B_{\rho}(b)} u d x\right)$, then $u$ is $\delta$-Hölder continuous in $B \frac{\rho}{2}(b)$ and $|u(x)-u(y)| \leq c|x-y|^{\delta}$ holds for any $x, y \in B_{\rho / 2}(b)$.

Proof. By Fubini's theorem there exists $\sigma \in[3 / 4 \rho, \rho]$ such that

$$
\begin{aligned}
\int_{\partial B_{\sigma}(b)}\left|u-(u)_{\rho, b}\right|^{2} d s & \leq \frac{8}{\rho} \int_{B_{\rho}(b)}\left|u-(u)_{\rho, b}\right|^{2} d x \leq 8 \epsilon_{0} \rho^{m-1} \\
\int_{\partial B_{\sigma}(b)}|\nabla u|^{2} d s & \leq \frac{8}{\rho} \int_{B_{\rho}(b)}|\nabla u|^{2} d x \leq 8 B \rho^{m-3} .
\end{aligned}
$$

By (3.1) and (3.2) we have

$$
\begin{aligned}
& \sigma^{4-2 n}\left(\int_{\partial B_{\sigma}(b)}\left|u-(u)_{\rho, b}\right|^{2} d s\right)\left(\int_{\partial B_{\sigma}(b)}|\nabla u|^{2} d s\right) \\
& \leq 64 B \epsilon_{0} \rho^{2 n-4} \sigma^{4-2 n} \leq \frac{245}{3} B \epsilon_{0} .
\end{aligned}
$$

Here we take $\epsilon_{0}>0$ such that $254 / 3 B \epsilon_{0}<\delta^{\prime 2} \epsilon^{q}$, where $\epsilon>0$ is a constant to be determined later and $\delta^{\prime}>0, q>0$ are constants appearing in the following extension lemma due to Schoen -Uhlenbeck (Lemma 4.3 in [13]):

Lemma 3.4 ([13, Lemma 4.3]). There exist $\delta^{\prime}>0$ and $q>0$ such that if $\epsilon \in(0,1)$ and $u \in H^{1}\left(\partial B_{\sigma}(b) ; N_{0}\right)$ satisfies

$$
\sigma^{4-2 m}\left(\int_{\partial B_{\sigma}(b)}\left|\nabla^{T} u\right|^{2} d s\right)\left(\int_{\partial B_{\sigma}(b)}|u-\xi|^{2} d s\right) \leq \delta^{\prime 2} \epsilon^{q}
$$

for some $\xi \in \mathbf{R}^{k}$, then there exists $\bar{u} \in H^{1}\left(B_{\sigma}(b) ; N_{0}\right)$ such that $\bar{u}=u$ on $\partial B_{\sigma}(b)$ and the following holds:

$$
\int_{B_{\sigma}(b)}|\nabla \bar{u}|^{2} d x \leq c\left(\epsilon \sigma \int_{\partial B_{\sigma}(b)}\left|\nabla^{T} u\right|^{2} d s+\epsilon^{-q} \sigma^{-1} \int_{\partial B_{\sigma}(b)}|u-\xi|^{2} d s\right) .
$$

By this lemma, there exists $\bar{u} \in H^{1}\left(B_{\sigma}(b) ; N_{0}\right)$ such that $\bar{u}=u$ on $\partial B_{\sigma}(b)$ and, by (3.1), (3.2),

$$
\int_{B_{\sigma}(b)}|\nabla \bar{u}|^{2} d x \leq c\left(8 \epsilon \sigma \rho^{m-3} B+8 \epsilon^{-q} \sigma^{-1} \epsilon_{0} \rho^{m-1}\right)
$$




$$
\begin{aligned}
& \leq c\left(8 B \epsilon \rho^{m-2}+\frac{32}{3} \epsilon_{0} \epsilon^{-q} \rho^{m-2}\right) \\
& \leq c\left(8 B \epsilon+\frac{32}{3} \epsilon_{0} \epsilon^{-q}\right) \rho^{m-2} .
\end{aligned}
$$

On the other hand, by Lemma 3.1, there exists $\eta \in H_{\varphi}^{1}\left(\Omega ; N_{0}\right)$ (depending on $\sigma, b, u$ ) such that

$$
\int_{B_{\sigma}(b)}|\nabla u|^{2} d x \leq \int_{B_{\sigma}(b)}|\nabla \bar{u}|^{2} d x+\beta_{\max }\left(\int_{B_{\sigma}(b)}|\nabla t(u)|^{2}+|\nabla t(\eta)|^{2} d x\right) .
$$

Combining (3.4) with (3.5) we obtain

$$
\begin{aligned}
\int_{B_{\sigma}(b)}|\nabla u|^{2} d x \leq & c\left(8 B \epsilon+\frac{32}{3} \epsilon_{0} \epsilon^{-q}\right) \rho^{m-2} \\
& +\beta_{\max }\left(\int_{B_{\sigma}(b)}|\nabla t(u)|^{2}+|\nabla t(\eta)|^{2} d x\right) .
\end{aligned}
$$

By De Giorgi-Nash theorem, there exist $c>0, \alpha>0$ (as in (2.19)) depending only on $\beta$ such that

$$
\sigma^{2-m} \int_{B_{\sigma}(b)}|\nabla t(u)|^{2} d x \leq c \sigma^{\alpha}, \quad \sigma^{2-m} \int_{B_{\sigma}(b)}|\nabla t(\eta)|^{2} d x \leq c \sigma^{\alpha} .
$$

Combining (3.6) and (3.7) we obtain

$$
\int_{B_{\sigma}(b)}|\nabla u|^{2} d x \leq c\left(8 B \epsilon+\frac{32}{3} \epsilon_{0} \epsilon^{-q}+\rho_{0}^{\alpha}\right) \rho^{m-2} .
$$

First we take $\epsilon>$ such that $c \cdot 8 B \epsilon<\bar{\epsilon} / 3$. Then choose $\epsilon_{0}>0$ such that $c \cdot 32 / 3 \epsilon_{0} \epsilon^{-q}<\bar{\epsilon} / 3$ (we may assume $254 / 3 B \epsilon_{0}<\delta^{\prime 2} \epsilon^{q}$ also holds). Finally choose $\rho_{0}>0$ such that $c \cdot \rho_{0}^{\alpha}<\bar{\epsilon} / 3$. Then for $\rho<\rho_{0}$, we have, by (3.8),

$$
\rho^{2-m} \int_{B_{\sigma}(b)}|\nabla u|^{2} d x \leq \bar{\epsilon} .
$$

Thus by Lemma 3.2, $u$ is Hölder continuous in $B_{\rho / 2}(b)$ and $|u(x)-u(y)| \leq$ $c|x-y|^{\delta}$ holds for some $c>0$ and $\delta>0$.

For $a \in \Omega$ and $\lambda>0$, define the scaled map $u_{\lambda, a}:=u(\lambda x+a)$.

Our next subject is to study the behavior of $u_{\lambda, a}$ for $\mathcal{F}$-minimizer $u$ as $\lambda \downarrow$ 0. For later purpose (see the proof of Corollary 3.6), we consider somewhat more general case. 
Let $a \in \Omega$ and $R<1 / 2 \operatorname{dist}(a, \partial \Omega)$. Let $u$ be a $\mathcal{F}$-minimizing map in $H_{\varphi}^{1}\left(M ; N_{0}\right)$. For $0<\lambda_{i} \leq 1, a_{i} \in B_{R}(a)$ with $\lambda_{i} \downarrow 0$ and $a_{i} \rightarrow a$, we consider the sequence $\left\{u_{\lambda_{i}, a_{i}}\right\}$.

Lemma 3.5. Let $u, \lambda_{i}$ and $a_{i}$ be as above. Then there exists a subsequence of $\left\{u_{\lambda_{i}, a_{i}}\right\}$ (which we also write $\left\{u_{\lambda_{i}, a_{i}}\right\}$ ) such that for any $r>0$ the following hold:

(a) $\quad u_{\lambda_{i}, a_{i}} \rightarrow u_{\infty}\left(\right.$ for some $\left.u_{\infty} \in H_{\mathrm{loc}}^{1}\left(\mathbf{R}^{m} ; N_{0}\right)\right)$ locally uniformly in $B_{r}(0) \backslash$ $\Sigma_{\infty}$, where $\Sigma_{\infty} \subset B_{r}(0)$ is a closed subset with $\mathcal{H}^{m-2}\left(\Sigma_{\infty}\right)=0$. Moreover $u_{\infty}$ is continuous in $B_{r}(0) \backslash \Sigma_{\infty}$.

(b) $u_{\lambda_{i}, a_{i}} \rightarrow u_{\infty}$ strongly in $H^{1}\left(B_{r}(0)\right)$.

(c) In particular if $a_{i} \equiv a$, then $\frac{\partial u_{\infty}}{\partial r}=0$.

Proof. (a) We fix $r>0$. For $i$ large we have $B_{r \lambda_{i}}\left(a_{i}\right) \subset \Omega$. We may assume without loss of generality that this hold for all $i$.

By Proposition 2.1, there exists $c>0$ independent of $i$ such that

$$
\int_{B_{r}(0)}\left|\nabla u_{\lambda_{i}, a_{i}}\right|^{2} d x=\lambda_{i}^{2-m} \int_{B_{r_{i}}\left(a_{i}\right)}|\nabla u|^{2} d x<c .
$$

Therefore there exists a subsequence of $\left\{u_{\lambda_{i}, a_{i}}\right\}$ (we also denote it as $\left\{u_{\lambda_{i}, a_{i}}\right\}$ ) such that

$$
\begin{array}{ccc}
u_{\lambda_{i}, a_{i}} \rightarrow u_{\infty} & \text { weakly in } & H^{1}\left(B_{r}(0)\right), \\
u_{\lambda_{i}, a_{i}} \rightarrow u_{\infty} & \text { strongly in } \quad L^{2}\left(B_{r}(0)\right) .
\end{array}
$$

For $b \in B_{r}(0)$ and $\rho<\operatorname{dist}\left(b, \partial B_{r}(0)\right)$ small we assume

$$
\rho^{-m} \int_{B_{\rho}(b)}\left|u_{\infty}-\left(u_{\infty}\right)_{\rho, b}\right|^{2} d x<\epsilon_{0},
$$

where $\epsilon_{0}>0$ is as in Proposition 3.3 for $B=c$.

Since $u_{\lambda_{i}, a_{i}} \rightarrow u_{\infty}$ in $L^{2}\left(B_{r}(0)\right)$, for $i$ large enough we have

$$
\rho^{-m} \int_{B_{\rho}(b)}\left|u_{\lambda_{i}, a_{i}}-\left(u_{\infty}\right)_{\rho, b}\right|^{2} d x<\epsilon_{0} .
$$

By the change of variable, we obtain from (3.11)

$$
\left(\lambda_{i} \rho\right)^{-m} \int_{B_{\lambda_{i} \rho}\left(a_{i}+\lambda_{i} b\right)}\left|u-\left(u_{\infty}\right)_{\rho, b}\right|^{2} d x<\epsilon_{0} .
$$

On the other hand, for $i$ large enough,

$$
\rho^{2-m} \int_{B_{\rho}(b)}\left|\nabla u_{\lambda_{i}, a_{i}}\right|^{2} d x=\left(\lambda_{i} \rho\right)^{2-m} \int_{B_{\lambda_{i} \rho}\left(a_{i}+\lambda_{i} b\right)}|\nabla u|^{2} d x \leq c .
$$


By Proposition 3.3, $u$ is $\delta$-Hölder continuous in $B_{\lambda_{i} \rho / 2}\left(a_{i}+\lambda_{i} b\right)$ and $\mid u(x)-$ $u(y)|\leq c| x-\left.y\right|^{\delta}$ for $x, y \in B_{\lambda_{i} \rho / 2}\left(a_{i}+\lambda_{i} b\right)$. By rescaling, $u_{\lambda_{i}, a_{i}}$ is $\delta$-Hölder continuous in $B_{\rho / 2}(b)$ and $\left|u_{\lambda_{i}, a_{i}}(x)-u_{\lambda_{i}, a_{i}}\right| \leq c \lambda_{i}^{\delta}|x-y|^{\delta} \leq c|x-y|^{\delta}$ for $x, y \in B_{\rho / 2}(b)$. Thus $\left\{u_{\lambda_{i}, a_{i}}\right\}$ is equi-continuous and equi-bounded in $B_{\rho}(b)$ and by Arzela-Ascoli's theorem, for some subsequence (we also denote it $\left.\left\{u_{\lambda_{i}, a_{i}}\right\}\right)$ such that

$$
u_{\lambda_{i}, a_{i}} \rightarrow u_{\infty} \quad \text { uniformly in } \quad B_{\frac{\rho}{2}}(b) .
$$

By Poincaré inequality, if $\rho^{2-m} \int_{B_{\rho}(b)}\left|\nabla u_{\infty}\right|^{2} d x$ is small enough, then (3.10) holds. So by the standard covering argument, there exists closed subset $\Sigma_{\infty}$ with $\mathcal{H}^{m-2}\left(\Sigma_{\infty}\right)=0$ such that

$$
u_{\lambda_{i}, a_{i}} \rightarrow u_{\infty} \text { locally uniformly in } B_{r}(0) \backslash \Sigma_{\infty} .
$$

Since $r>0$ is arbitrary, by diagonal sequence argument, we obtain (a).

(b) We fix $r>0$ as in (a). We prove $u_{\lambda_{i}, a_{i}} \rightarrow u_{\infty}$ strongly in $H^{1}\left(B_{r}(0)\right)$.

Let $\Sigma_{\infty}$ be as in part (a). Since $\mathcal{H}^{m-2}\left(\Sigma_{\infty} \cap B_{r}(0)\right)=0$, for any $\epsilon>0$, there exists balls $\left\{B_{r_{i}}\left(x_{i}\right)\right\}$ such that $\sum_{i} r_{i}^{m-2}<\epsilon, \Sigma_{\infty} \cap B_{r}(0) \subset \bigcup_{i} B_{r_{i}}\left(x_{i}\right)$, $x_{i} \in B_{r}(0)$.

By Proposition 2.1, there exists $c>0$ independent of $k$ and $i$ such that

$$
r_{i}^{2-m} \int_{B_{r_{i}}\left(x_{i}\right)}\left|\nabla u_{\lambda_{k}, a_{k}}\right|^{2} d x=\left(\lambda_{k} r_{i}\right)^{2-m} \int_{B_{\lambda_{k} r_{i}}\left(a_{k}+\lambda_{k} x_{i}\right)}|\nabla u|^{2} d x \leq c .
$$

Therefore we have

$$
\sum_{i} \int_{B_{r_{i}}\left(x_{i}\right)}\left|\nabla u_{\lambda_{k}, a_{k}}\right|^{2} d x \leq c \sum_{i} r_{i}^{m-2}<c \epsilon
$$

for all large $k$.

On the other hand, by the first equation of (1.1), we obtain

$$
\begin{aligned}
\Delta u_{\lambda_{k}, a_{k}}+A\left(u_{\lambda_{k}, a_{k}}\right)\left(\nabla u_{\lambda_{k}, a_{k}},\right. & \left.\nabla u_{\lambda_{k}, a_{k}}\right) \\
+ & \frac{1}{2} \nabla \beta\left(u_{\lambda_{k}, a_{k}}\right)\left|\nabla t(u)_{\lambda_{k}, a_{k}}\right|^{2}=0 \quad \text { in } \quad B_{r}(0) .
\end{aligned}
$$

Here $t(u)_{\lambda_{k}, a_{k}}(x)=t(u)\left(\lambda_{k} x+a_{k}\right)$.

Thus we have

$$
\begin{aligned}
& \Delta\left(u_{\lambda_{k}, a_{k}}-u_{\lambda_{j}, a_{j}}\right) \\
& =-A\left(u_{\lambda_{k}, a_{k}}\right)\left(\nabla u_{\lambda_{k}, a_{k}}, \nabla u_{\lambda_{k}, a_{k}}\right)+A\left(u_{\lambda_{j}, a_{j}}\right)\left(\nabla u_{\lambda_{j}, a_{j}}, \nabla u_{\lambda_{j}, a_{j}}\right) \\
& \quad-\frac{1}{2} \nabla \beta\left(u_{\lambda_{k}, a_{k}}\right)\left|\nabla t(u)_{\lambda_{k}, a_{k}}\right|^{2}+\frac{1}{2} \nabla \beta\left(u_{\lambda_{j}, a_{j}}\right)\left|\nabla t(u)_{\lambda_{j}, a_{j}}\right|^{2} .
\end{aligned}
$$


Take $\varphi \in C_{0}^{\infty}\left(B_{r}(0) \backslash \bigcup_{i} B_{r_{i}}\left(x_{i}\right)\right)$ arbitrary. Multiplying $\varphi\left(u_{\lambda_{k}, a_{k}}-u_{\lambda_{j}, a_{j}}\right)$ by (3.14) and integrating over $B_{r}(0)$, we obtain

$$
\begin{aligned}
& \int_{B_{r}(0)} \varphi\left|\nabla u_{\lambda_{k}, a_{k}}-\nabla u_{\lambda_{j}, a_{j}}\right|^{2} d x \\
& \leq c \int_{B_{r}(0)}|\varphi|\left(\left|\nabla u_{\lambda_{k}, a_{k}}\right|^{2}+\left|\nabla u_{\lambda_{j}, a_{j}}\right|^{2}\right)\left|u_{\lambda_{k}, a_{k}}-u_{\lambda_{j}, a_{j}}\right| d x \\
& \quad+c \int_{B_{r}(0)}|\varphi|\left(\left|\nabla t(u)_{\lambda_{k}, a_{k}}\right|^{2}+\left|\nabla t(u)_{\lambda_{j}, a_{j}}\right|^{2}\right)\left|u_{\lambda_{k}, a_{k}}-u_{\lambda_{j}, a_{j}}\right| d x \\
& \quad+c \int_{B_{r}(0)}|\nabla \varphi|\left|\nabla u_{\lambda_{k}, a_{k}}-\nabla u_{\lambda_{j}, a_{j}}\right|\left|u_{\lambda_{k}, a_{k}}-u_{\lambda_{j}, a_{j}}\right| d x .
\end{aligned}
$$

Here, by Proposition 2.1 and De Giorgi-Nash theorem, there exists $c>0$ independent of $k$ such that

$$
\begin{gathered}
\int_{B_{r}(0)}\left|\nabla u_{\lambda_{k}, a_{k}}\right|^{2} d x=\lambda_{k}^{2-m} \int_{B_{r_{k}}\left(a_{k}\right)}|\nabla u|^{2} d x \leq c \\
\int_{B_{r}(0)}\left|\nabla t(u)_{\lambda_{k}, a_{k}}\right|^{2} d x=\lambda_{k}^{2-m} \int_{B_{r_{\lambda_{k}}\left(a_{k}\right)}|\nabla t(u)|^{2} d x \leq c .}
\end{gathered}
$$

Combining (3.13), (3.15), (3.16) and (3.17), and since $\varphi \in C_{0}^{\infty}\left(B_{r}(0) \backslash\right.$ $\left.\bigcup_{i} B_{r_{i}}\left(x_{i}\right)\right)$ is arbitrary, we obtain, for some subsequence of $\left\{u_{\lambda_{i}, a_{i}}\right\}$ (we also denote it by $\left\{u_{\lambda_{i}, a_{i}}\right\}$ )

$$
u_{\lambda_{i}, a_{i}} \rightarrow u_{\infty} \text { strongly in } H^{1}\left(B_{r}(0)\right) .
$$

Since $r>0$ is arbitrary, by diagonal sequence argument, we obtain the result.

(c) By Proposition 2.1, we have, for $j \leq i$

$$
\begin{aligned}
& \lambda_{i}^{2-m} \int_{B_{\lambda_{i}}(a)}|\nabla u|^{2} d x+2 \int_{\lambda_{i} \leq|x-a| \leq \lambda_{j}}|x-a|^{2-m}\left|\frac{\partial u}{\partial r}\right|^{2} d x \\
& \leq \lambda_{j}^{2-m} \int_{B_{\lambda_{j}}(a)}|\nabla u|^{2} d x+c\left(\lambda_{i}^{\alpha}+\lambda_{j}^{\alpha}\right) .
\end{aligned}
$$

By Proposition 2.1, there exists limit $\lim _{r \downarrow 0} r^{2-m} \int_{B_{r}(a)}|\nabla u|^{2} d x=L$. Letting $i \rightarrow \infty$ in (3.18), we obtain

$$
L+2 \int_{|x-a| \leq \lambda_{j}}|x-a|^{2-m}\left|\frac{\partial u}{\partial r}\right|^{2} d x \leq \lambda_{j}^{2-m} \int_{B_{\lambda_{j}}(a)}|\nabla u|^{2} d x+c \lambda_{j}^{\alpha} .
$$


By rescaling, we obtain

$$
L+2 \int_{|x| \leq 1}|x|^{2-m}\left|\frac{\partial u_{\lambda_{j}, a}}{\partial r}\right|^{2} d x \leq \lambda_{j}^{2-m} \int_{B_{\lambda_{j}}(a)}|\nabla u|^{2} d x+c \lambda_{j}^{\alpha} .
$$

Since $u_{\lambda_{j}, a} \rightarrow u_{\infty}$ strongly in $H_{\mathrm{loc}}^{1}\left(\mathbf{R}^{m} ; N_{0}\right)$, taking a limit $j \rightarrow \infty$ in (3.20), we obtain

$$
\int_{|x| \leq 1}|x|^{2-m}\left|\frac{\partial u_{\infty}}{\partial r}\right|^{2} d x=0
$$

Therefore $\frac{\partial u_{\infty}}{\partial r}=0$.

\section{Corollary 3.6.}

(i) The conclusions of Lemma 3.5 hold for maps in

$\mathcal{T} \mathcal{M}=\left\{v:\right.$ there exist $\lambda_{i} \downarrow 0, a_{i} \rightarrow$ a such that $u_{\lambda_{i}, a_{i}} \rightarrow v$ in $\left.H_{\mathrm{loc}}^{1}\left(\mathbf{R}^{m}\right)\right\}$.

Here $a \in \Omega$ and $u$ is a $\mathcal{F}$-minimizing map in $H_{\varphi}^{1}\left(\Omega ; N_{0}\right)$.

(ii) Define $\mathcal{T} \mathcal{M}^{l}(l \geq 0)$ inductively as follows: $\mathcal{T} \mathcal{M}^{0}=\mathcal{T} \mathcal{M}, \mathcal{T} \mathcal{M}^{l}=\{v$ : there exist $u \in \mathcal{T} \mathcal{M}^{l-1}, \lambda_{i} \downarrow 0$ and $a_{i} \rightarrow a \in \mathbf{R}^{m}$ such that $u_{\lambda_{i}, a_{i}} \rightarrow v$ in $\left.H_{\mathrm{loc}}^{1}\left(\mathbf{R}^{m}\right)\right\}$ for $l \geq 1$. Then the conclusions of Lemma 3.5 hold for maps in $\mathcal{T M}^{l}$ for $l \geq 0$.

Proof. (i) First we show the monotonicity property for the maps in $\mathcal{T} \mathcal{M}$. Let $v \in \mathcal{T} \mathcal{M}, b \in \mathbf{R}^{m}$ and $0<R_{1}<R_{2}$. Then there exist sequences $\left\{\lambda_{i}\right\}, \lambda_{i} \downarrow 0$ and $\left\{a_{i}\right\}, a_{i} \rightarrow a$ such that $u_{\lambda_{i}, a_{i}} \rightarrow v$ in $H_{\mathrm{loc}}^{1}\left(\mathbf{R}^{m}\right)$. By Proposition 2.1, we have

$$
\begin{aligned}
& \left(\lambda_{i} R_{1}\right)^{2-m} \int_{B_{\lambda_{i} R_{1}}\left(a_{i}+\lambda_{i} b\right)}|\nabla u|^{2} d x \\
& \quad+\int_{\lambda_{i} R_{1} \leq\left|x-a_{i}-\lambda_{i} b\right| \leq \lambda_{i} R_{2}}\left|x-a_{i}-\lambda_{i} b\right|^{2-m}\left|\frac{\partial u}{\partial r}\right|^{2} d x \\
& \leq\left(\lambda_{i} R_{2}\right)^{2-m} \int_{B_{\lambda_{i} R_{2}}\left(a_{i}+\lambda_{i} b\right)}|\nabla u|^{2} d x+c\left(\left(\lambda_{i} R_{1}\right)^{\alpha}+\left(\lambda_{i} R_{2}\right)^{\alpha}\right) .
\end{aligned}
$$

From this, we obtain:

$$
\begin{aligned}
& R_{1}^{2-m} \int_{B_{R_{1}}(b)}\left|\nabla u_{\lambda_{i}, a_{i}}\right|^{2} d x+2 \int_{R_{1} \leq|x-b| \leq R_{2}}|x-b|^{2-m}\left|\frac{\partial u_{\lambda_{i}, a_{i}}}{\partial r}\right|^{2} d x \\
& \leq R_{2}^{2-m} \int_{B_{R_{2}}(b)}\left|\nabla u_{\lambda_{i}, a_{i}}\right|^{2} d x+c\left(\left(\lambda_{i} R_{1}\right)^{\alpha}+\left(\lambda_{i} R_{2}\right)^{\alpha}\right) .
\end{aligned}
$$


Letting $i \rightarrow \infty$, we obtain the following monotonicity inequality:

$$
\begin{aligned}
R_{1}^{2-m} \int_{B_{R_{1}}(b)}|\nabla v|^{2} d x+2 \int_{R_{1} \leq|x-b| \leq R_{2}}|x-b|^{2-m} & \left|\frac{\partial v}{\partial r}\right|^{2} d x \\
& \leq R_{2}^{2-m} \int_{B_{R_{2}}(b)}|\nabla v|^{2} d x .
\end{aligned}
$$

Next observe that for $v \in \mathcal{T} \mathcal{M}, \lambda>0$ and $b \in B_{1}(0)$ we have $v_{\lambda, b} \in \mathcal{T} \mathcal{M}$ since $\left(u_{\lambda_{i}, a_{i}}\right)_{\lambda, b}=u_{\lambda_{i} \lambda, \lambda_{i} b+a_{i}}$. (Note that even in the case $a_{i} \equiv a, \lambda_{i} b+a_{i}=$ $\lambda_{i} b+a \neq a$. For this reason, we have considered the case $a_{i} \rightarrow a$ and not the case $a_{i} \equiv a$.)

We first prove the assertion (a) for the map $v \in \mathcal{T} \mathcal{M}$. Fix $r>0$. Let $v \in \mathcal{T} \mathcal{M}$ as above, $\left\{\lambda_{i}^{\prime}\right\}, \lambda_{i}^{\prime} \downarrow 0$ and $a_{i}^{\prime} \rightarrow a^{\prime}$. Consider the rescaled maps $v_{\lambda_{i}^{\prime}, a_{i}^{\prime}}$.

By the monotonicity inequality for the maps in $\mathcal{T} \mathcal{M}$ proved above, there exists subsequence of $\left\{v_{\lambda_{i}^{\prime}, a_{i}^{\prime}}\right\}$ (we also denote it by $\left\{v_{\lambda_{i}^{\prime}, a_{i}^{\prime}}\right\}$ ) such that $v_{\lambda_{i}^{\prime}, a_{i}^{\prime}} \rightarrow$ $v_{\infty}$ weakly in $H^{1}\left(B_{r}(0)\right)$ and $v_{\lambda_{i}^{\prime}, a_{i}^{\prime}} \rightarrow v_{\infty}$ strongly in $L^{2}\left(B_{r}(0)\right)$.

Let $\zeta \in B_{r}(0)$ and $\rho<\operatorname{dist}\left(\zeta, \partial B_{r}(0)\right)$; small be such that $\rho^{-m} \int_{B_{\rho}(\zeta)} \mid v_{\infty}-$ $\left.\left(v_{\infty}\right)_{\rho, \zeta}\right|^{2} d x<\epsilon_{0}$. Then, as in the proof of Lemma 3.5 (a), there exists $i_{0}$ such that for $i \geq i_{0}$, we have

$$
\rho^{-m} \int_{B_{\rho}(\zeta)}\left|v_{\lambda_{i}^{\prime}, a_{i}^{\prime}}-\left(v_{\infty}\right)_{\rho, \zeta}\right|^{2}<\epsilon_{0}
$$

Since $u_{\lambda_{k}, a_{k}} \rightarrow v$ in $H_{\mathrm{loc}}^{1}\left(\mathbf{R}^{m}\right)$, there exists $k(i)$ such that for $k \geq k(i)$

$$
\rho^{-m} \int_{B_{\rho}(\zeta)}\left|\left(u_{\lambda_{k}, a_{k}}\right)_{\lambda_{i}^{\prime}, a_{i}^{\prime}}-\left(v_{\infty}\right)_{\rho, \zeta}\right|^{2} d x<\epsilon_{0}
$$

Since $\left(u_{\lambda_{k}, a_{k}}\right)_{\lambda_{i}^{\prime}, a_{i}^{\prime}}=u_{\lambda_{k} \lambda_{i}^{\prime}, \lambda_{k} a_{i}^{\prime}+a_{k}}$, we get

$$
\left(\lambda_{k} \lambda_{i}^{\prime} \rho\right)^{-m} \int_{B_{\lambda_{k} \lambda_{i}^{\prime} \rho}\left(\lambda_{k} a_{i}^{\prime}+a_{k}+\lambda_{k} \lambda_{i}^{\prime} \zeta\right)}\left|u-\left(v_{\infty}\right)_{\rho, \zeta}\right|^{2} d x<\epsilon_{0}
$$

for such $i$ and $k$.

Then by the same argument in the proof of Lemma 3.5 (a), $u$ is Hölder continuous in $B_{\lambda_{k} \lambda_{i}^{\prime} \rho / 2}\left(\lambda_{k} a_{i}^{\prime}+a_{k}+\lambda_{k} \lambda_{i}^{\prime} \zeta\right)$, and there exists constant $c>0$ such that $\left|\left(u_{\lambda_{k}, a_{k}}\right)_{\lambda_{i}^{\prime}, a_{i}^{\prime}}(x)-\left(u_{\lambda_{k}, a_{k}}\right)_{\lambda_{i}^{\prime}, a_{i}^{\prime}}(y)\right| \leq c|x-y|^{\delta}$ for $x, y \in B_{\rho / 2}(\zeta)$. From this, $\left\{\left(u_{\lambda_{k}, a_{k}}\right)_{\lambda_{i}^{\prime}, a_{i}^{\prime}}\right\}_{k \geq k(i)}$ is equi-continuous and, by Arzela-Ascoli, there exists a subsequence (we also denote it by the same sequence) such that $\left(u_{\lambda_{k}, a_{k}}\right)_{\lambda_{i}^{\prime}, a_{i}^{\prime}} \rightarrow v_{i}$ uniformly in $B_{\rho / 2}(\zeta)$ for some $v_{i}$ as $k \rightarrow \infty$. But $u_{\lambda_{k}, a_{k}} \rightarrow v$ in $H_{\text {loc }}^{1}\left(\mathbf{R}^{m}\right)$, so we have $v_{i} \equiv v_{\lambda_{i}^{\prime}, a_{i}^{\prime}}$. By the uniform convergence, $v_{\lambda_{i}^{\prime}, a_{i}^{\prime}}$ also satisfies $\left|v_{\lambda_{i}^{\prime}, a_{i}^{\prime}}(x)-v_{\lambda_{i}^{\prime}, a_{i}^{\prime}}(y)\right| \leq c|x-y|^{\delta}$ for $x, y \in B_{\rho / 2}(\zeta)$ and $i \geq i_{0}$. Again 
by Arzela-Ascoli, we have $v_{\lambda_{i}^{\prime}, a_{i}^{\prime}} \rightarrow v_{\infty}$ uniformly in $B_{\rho / 2}(\zeta)$. From this and the same argument in the proof of Lemma 3.5 (a) shows that the conclusion of Lemma 3.5 (a) also holds for maps in $\mathcal{T} \mathcal{M}$.

Next we verify Lemma 3.5 (b) for maps in $\mathcal{T} \mathcal{M}$. For this, first observe that the map $v \in \mathcal{T} \mathcal{M}$ is a harmonic map into $N_{0}$, that is, it satisfies the equation $\Delta v+A(v)(\nabla v, \nabla v)=0$. To see this, we only note that the left hand side of (3.17) goes to zero as $k \rightarrow \infty$ by De Giorgi-Nash theorem. Then, by the result (a), the same argument in the proof of Lemma 3.5 (b) shows that the conclusion (b) of Lemma 3.5 also holds for maps in $\mathcal{T} \mathcal{M}$.

The proof of Lemma 3.5 (c) for maps in $\mathcal{T} \mathcal{M}$ follows the same argument in the proof of Lemma 3.5 (c), since we already have monotonicity inequality, (a) and (b).

(ii) The proof of assertion (ii) follows from the same argument in (i) using the induction on $l$.

Remark 3.7. (a) As was stated in the introduction, $\mathcal{F}$-minimizing map in $H_{\varphi}^{1}\left(M ; N_{0}\right)$ does not have local minimizing property. So there is no sense to consider local minimizing maps to study regularity properties of $\mathcal{F}$-minimizing maps and, of course, scaled maps $u_{\lambda, a}$ for $\mathcal{F}$-minimizing map $u$ does not have any local minimizing property. Therefore in the above proposition and corollary, we only considered the compactness properties of scaled maps (not local minimizing maps as in many other problems). This is the troublesome point in our problem.

(b) The limiting map $v \in \mathcal{T} \mathcal{M}$, which we call tangent map at $a$ in the case $a_{i} \equiv a$, is indeed a harmonic map into $N_{0}$. This follows from the same reason as in Corollary 3.6 (i), proof of part (b).

We are now ready to prove our main theorem Theorem A. As in many other regularity problems, we prove it by Federer's dimension reduction method [3], [12], [13].

Completion of the proof of Theorem A. We define the measure $\varphi^{s}$ for $s \in \mathbf{R}$ as follows:

$$
\varphi^{s}(E)=\inf \left\{\sum_{i} r_{i}^{s}: E \subset \bigcup_{i=1}^{\infty} B_{r_{i}}\left(x_{i}\right)\right\} .
$$

We prove the following

Lemma 3.8. Let $u$ be $\mathcal{F}$-minimizing map or $u \in \mathcal{T} \mathcal{M}^{l}, l \geq 0$. Let $\lambda_{i} \downarrow 0$ and $a \in B_{1}(0)$. Assume $u_{\lambda_{i}, a} \rightarrow u_{\infty}$ weakly in $H^{1}\left(B_{1} ; N_{0}\right)$. Let $\Sigma_{i}$ and $\Sigma_{\infty}$ be singular sets of $u_{\lambda_{i}, a}$ and $u_{\infty}$, respectively. Then we have

$$
\varphi^{s}\left(\Sigma_{\infty} \cap B_{1 / 2}(0)\right) \geq \limsup _{i \rightarrow \infty} \varphi^{s}\left(\Sigma_{i} \cap B_{1 / 2}(0)\right) .
$$


Proof. Let $\epsilon>0$ be given. Let $\left\{B_{r_{i}}\left(x_{i}\right)\right\}$ be a sequence of balls such that $\Sigma_{\infty} \cap B_{1 / 2}(0) \subset \bigcup_{i=1}^{\infty} B_{r_{i}}\left(x_{i}\right)$ and

$$
\sum_{i=1}^{\infty} r_{i}^{s} \leq \varphi^{s}\left(\Sigma_{\infty} \cap B_{1 / 2}(0)\right)+\epsilon
$$

By Lemma 3.5 and Corollary 3.6, for large $i, u_{\lambda_{i}, a}$ is continuous on $B_{1 / 2}(0) \backslash$ $\bigcup_{i=1}^{\infty} B_{r_{i}}\left(x_{i}\right)$. Therefore we have

$$
\Sigma_{i} \cap B_{1 / 2}(0) \subset \bigcup_{i=1}^{\infty} B_{r_{i}}\left(x_{i}\right)
$$

So

$$
\varphi^{s}\left(\Sigma_{i} \cap B_{1 / 2}(0)\right) \leq \sum_{i=1}^{\infty} r_{i}^{s} \leq \varphi^{s}\left(\Sigma_{\infty} \cap B_{1 / 2}(0)\right)+\epsilon .
$$

Since $\epsilon>0$ is arbitrary, we obtain the result.

We continue the proof of Theorem A. Let $\Sigma$ be a singular set of $u$. By Theorem 1.1, we know $\mathcal{H}^{m-2}(\Sigma)=0$.

Let $0 \leq s<m-2$ be a real number such that $\varphi^{s}(\Sigma)>0$. If there is no such $s$, then we complete the proof, since in this case $\Sigma=\emptyset$.

By the density theorem about the measure $\varphi^{s}$, see [12], we have $\lim \sup _{\lambda \downarrow 0} \lambda^{-s} \varphi^{s}\left(\Sigma \cap B_{\lambda / 2}(x)\right)>0$ for $\varphi^{s}$-a.e. $x \in \Sigma$. So there exist $a_{0} \in \Sigma$ and $\lambda_{i} \downarrow 0$ such that

$$
\lim _{i \rightarrow \infty} \lambda_{i}^{-s} \varphi^{s}\left(\Sigma \cap B_{\lambda_{i} / 2}\left(a_{0}\right)\right)>0
$$

We consider the rescaled maps $u_{\lambda_{i}, a_{0}}$.

By Lemma 3.5, there exists a subsequence (we also denote it by $\left\{u_{\lambda_{i}, a_{0}}\right\}$ ) such that

$$
u_{\lambda_{i}, a_{0}} \rightarrow u_{0} \quad \text { strongly in } \quad H_{\mathrm{loc}}^{1}\left(\mathbf{R}^{m} ; N_{0}\right)
$$

for some $u_{0} \in H_{\text {loc }}^{1}\left(\mathbf{R} ; N_{0}\right)$ with $\frac{\partial u_{0}}{\partial r}=0$.

We set $\Sigma_{i}^{a_{0}}=$ the singular set of $u_{\lambda_{i}, a_{0}}$. Then $\varphi^{s}\left(\Sigma_{i}^{a_{0}} \cap B_{1 / 2}(0)\right)=$ $\lambda_{i}^{-s} \varphi^{s}\left(\Sigma \cap B_{\lambda_{i} / 2}\left(a_{0}\right)\right)$ and $\lim _{i \rightarrow \infty} \varphi^{s}\left(\Sigma_{i}^{a_{0}} \cap B_{1 / 2}(0)\right)>0$.

By Lemma 3.8, we have $\varphi^{s}\left(\Sigma_{0} \cap B_{1 / 2}(0)\right)>0$, where $\Sigma_{0}$ is the singular set of $u_{0}$. Since $\frac{\partial u_{0}}{\partial r}=0, \Sigma_{0}$ is a cone, i.e., $\lambda \Sigma=\Sigma$ for all $\lambda>0$.

There are two possibilities:

(i) $s=0$

(ii) $s>0$.

If the case (i) occurs, we complete the proof. So we consider the case (ii). We take a new coordinate so that the radial coordinate $r$ is $x_{1}$-direction. 
There exists $a_{1} \in \Sigma_{0} \cap \partial B_{1}(0)$ such that

$$
\limsup _{\lambda \downarrow 0} \lambda^{-s} \varphi^{s}\left(\Sigma_{0} \cap B_{\lambda}\left(a_{1}\right)\right)>0 .
$$

Then by the same argument as before (using Corollary 3.6), there exists a sequence $\left\{\lambda_{i}\right\}, \lambda_{i} \downarrow 0$ such that

$$
\lim _{i \rightarrow \infty} \lambda_{i}^{-s} \varphi^{s}\left(\Sigma_{0} \cap B_{\lambda_{i}}\left(a_{1}\right)\right)>0
$$

and for some subsequence of $\left\{u_{0 \lambda_{i}, x_{1}}\right\}$ (we also write it as $\left\{u_{\lambda_{i}, a_{1}}\right\}$ ) we have $u_{0 \lambda_{i}, a_{1}} \rightarrow u_{1}$ strongly in $H_{\text {loc }}^{1}\left(\mathbf{R}^{m} ; N_{0}\right)$, where $u_{1} \in \mathcal{T} \mathcal{M}^{1}$ and $\frac{\partial u_{1}}{\partial r_{1}}=0$. (Here $r_{1}$ is the radial with respect to the new coordinate.)

Let $\Sigma_{1}$ be a singular set of $u_{1}$. Then by Lemma 3.8, $\varphi^{s}\left(\Sigma_{1} \cap B_{1}(0)\right)>0$. Since $\frac{\partial u_{0}}{\partial x_{1}}=0$, we also have $\frac{\partial u_{1}}{\partial x_{1}}=0$.

If $s-1 \leq 0$, we stop. Otherwise (this is the case $s>1$ ), since $\varphi^{s}\left(\Sigma_{1} \cap\right.$ $\left.B_{1}(0) \backslash \mathbf{R} \times\{0\}\right)=\varphi^{s}\left(\Sigma_{1} \cap B_{1}(0)\right)>0$, there exists $a_{2} \in \Sigma_{2} \cap \partial B_{1}^{m-1}(0)$, where $B_{1}^{m-1}(0)$ is the unit ball in $\mathbf{R}^{m-1}=\left\{\left(0, x_{2}, \ldots, x_{m}\right)\right\}$, such that

$$
\limsup _{\lambda \downarrow 0} \lambda^{-s} \varphi^{s}\left(\Sigma_{1} \cap B_{\lambda}\left(a_{2}\right)\right)>0
$$

and there exists $u_{2} \in \mathcal{T} \mathcal{M}^{2}$ such that $\frac{\partial u_{2}}{\partial x_{1}}=\frac{\partial u_{2}}{\partial x_{2}}=\frac{\partial u_{2}}{\partial r}=0$ (for some suitable choice of coordinate).

We continue this procedure $n$-times. Then we have $u_{l} \in H_{\mathrm{loc}}^{1}\left(\mathbf{R}^{m} ; N_{0}\right) \cap$ $\mathcal{T} \mathcal{M}^{l}(1 \leq l \leq n)$ with $\frac{\partial u_{l}}{\partial x_{i}}=\frac{\partial u_{l}}{\partial r}=0$ for $i=1, \ldots, l$ (for suitable choice of coordinate). We can repeat the argument until we have $s-n \leq 0$.

In order to obtain constructed $u_{n}$, it is necessary $s-n+1>0$. Since $s<m-2$, we obtain $m-1>n$, i.e., $n \leq m-2$.

If $n=m-2$, then $\Sigma_{n}=$ singular set of $u_{n} \supset \mathbf{R}^{m-2}=\left\{\left(x^{1}, \ldots, x^{m-2}, 0,0\right)\right\}$. This is a contradiction since $\mathcal{H}^{m-2}\left(\Sigma_{n}\right)=0$. Therefore we have $n \leq m-3$ and $s \leq n \leq m-3$. Since $s$ is an any number satisfying $s<\operatorname{dim} \Sigma$, we obtain $\operatorname{dim} \Sigma \leq m-3$.

Finally, we consider the case $m=3$. We assume that there exists a limit point $x_{0} \in B_{r}\left(x_{0}\right) \subset \subset \Omega$ of $\Sigma$, that is, there exist distinct points $x_{i} \in \Sigma \cap B_{r}\left(x_{0}\right)$ such that $x_{i} \rightarrow x_{0}$ as $i \rightarrow \infty$. Put $\lambda_{i}=\left|x_{i}-x_{0}\right|$ and consider the sequence $\left\{u_{\lambda_{i}, x_{0}}\right\}$. We remark that singular set of $u_{\lambda_{i}, x_{0}} \cap \partial B_{1}(0) \neq \emptyset$. By Lemma 3.5, we may assume $u_{\lambda_{i}, x_{0}} \rightarrow u_{\infty}$ strongly in $H_{\text {loc }}^{1}\left(\mathbf{R}^{m} ; N_{0}\right)$ for some $u_{\infty} \in \mathcal{T} \mathcal{M}$. We may also assume that $x_{i}-x_{0} /\left|x_{i}-x_{0}\right| \rightarrow \zeta \in \partial B_{1}(0)$. Since $x_{i}$ is a singularity of $u$, by Lemma 3.2, we have

$$
\frac{1}{\lambda_{i} r} \int_{B_{\lambda_{i} r}\left(x_{i}\right)}|\nabla u|^{2} d x \geq \bar{\epsilon}
$$


and, by rescaling

$$
\frac{1}{r} \int_{B_{r}\left(\frac{x_{i}-x_{0}}{\left|x_{i}-x_{0}\right|}\right)}\left|\nabla u_{\lambda_{i}, x_{0}}\right|^{2} d x \geq \bar{\epsilon}>0
$$

for all small $r>0$.

Letting $i \rightarrow \infty$, we obtain

$$
\frac{1}{r} \int_{B_{r}(\zeta)}\left|\nabla u_{\infty}\right|^{2} d x \geq \bar{\epsilon}
$$

for all small $r>0$.

Therefore $\zeta$ is a singularity of $u_{\infty}$. Since $\Sigma_{\infty}\left(=\operatorname{singular}\right.$ set of $\left.u_{\infty}\right)$ is a cone, we have $\mathcal{H}^{1}\left(\Sigma_{\infty}\right)>0$. This is a contradiction since $\mathcal{H}^{1}\left(\Sigma_{\infty}\right)=0$. Therefore $\Sigma$ is a discrete set in the case $m=3$.

\section{References}

[1] F. Bethuel, On the singular set of stationary harmonic maps, Manuscripta Math., 78 (1993), 217-243.

[2] H. Brezis, J.M. Coron and E. Lieb, Harmonic maps with defect, Comm. Math. Phys., 107 (1986), 649-705.

[3] H. Federer, The singular set of area minimizing rectifiable currents with codimension one and of area minimizing flat chains modulo two with arbitrary codimension, Bull. A.M.S., 76 (1970), 767-771.

[4] C. Greco, The Dirichlet problem for harmonic maps from the disk into a lorentzian warped product, Ann. Inst. Henri Poincaré, Analyse nonlinéaire, 10 (1993), 239252.

[5] F. Hélein, Regularite des applications faiblement harmoniques entre une surface et une sphere, C.R. Acad. Sci. Paris, 312(I) (1991), 591-596.

[6] Legularity of weakly harmonic maps from surface into a manifold with symmetries, Manuscripta Math., 70 (1991), 203-218.

[7]_L Regularite des applications faiblement harmoniques entre surface et une variete riemannienne, C.R. Acad. Sci. Paris, 312(I) (1991), 591-596.

[8] T. Isobe, Regularity of harmonic maps into a static Lorentzian manifold, to appear in J. Geom. Anal.

[9] O. Ladyzhenskaya and N. Ural'tseva, Linear and Quasilinear Elliptic Equations, Academic Press, 1968.

[10] A. Masiello, Variational methods in Lorentzian geometry, Longman Scientific \& Technical, 309 (1994).

[11] T. Riviere, Everywhere discontinuous harmonic maps from the dimension 3 into spheres, preprint, CMLA, No 9302.

[12] L. Simon, Lectures on Geometric Measure Theory, Proc. Centre. Math. Anal., 3 (1983). 
[13] R. Schoen and K. Uhlenbeck, A regularity theory for harmonic maps, J. Diff. Geom., 17 (1982), 307-335.

Received June 8, 1995 and revised October 2, 1995.

Tokyo Institute of Technology

OH-OKAYAMA, MEgURO-KU

TOKYO 152, JAPAN

E-mail address: isobe@math.titech.ac.jp 